\title{
Health-Related Quality of Life of Diabetes Mellitus Patients and Non- Diabetic Persons in Calabar, Cross River State, Nigeria
}

\author{
Article by Iquo Okon \\ Doctor of Philosophy Degree, Public Health, Texilla American University \\ E-mail:iquokon@gmail.com
}

\begin{abstract}
Quality of life of persons living with diabetes measures their health status with a look at multiple aspects of their life, including: emotional reactions to life occurrences, disposition, sense of life fulfilment and satisfaction in life. This study compares Health related quality of life of persons living with diabetes and those without Diabetes in University of Calabar Teaching Hospital, Calabar. This is a facility based cross sectional descriptive study. Participants were given the World Health Organization quality of life (WHOQOL-BREF) to assess their quality of life. Data were analyzed using Statistical Packages for the Social Sciences (SPSS), version 24. The results were presented in Means, standard deviations, and statistical analyses were performed to compare between $Q O L$ between diabetic and non-diabetics. A total 400 respondents (200 people living with Diabetes and 200 people without diabetes) participated in this study. Clinical data of diabetic respondents showed that 70.5 percent $(n=141)$ were living with other disease while 29.5 percent $n=59)$ of diabetic responds were not living with other diseases. Diseases mostly exhibited by diabetic patients were hypertension 40 percent $(n=80)$ and arthritis 15 percent $(n=30)$. Patients with diabetes had a significant lower mean score than the non-diabetics in their overall quality of life $(P=0.000)$. Findings from this study also indicate that diabetics had a mean score of 23.17, 20.06, 10.20, 28.0 in their physical, psychological, social and environmental domain respectively compare with those without diabetes that reported a mean score of 24.17, 21.53, 11.43, 28.68 in their physical, psychological, social and environmental domain respectively. There is need for comprehensive management plan to minimize the daunting outcomes of DM and improve the QOL of the diabetics.
\end{abstract}

\section{Introduction}

Diabetes mellitus (DM) is a group of metabolic diseases characterized by hyperglycemia, from defects in insulin secretion. Diabetes affects more than 425 million adults have diabetes worldwide. Over 1 million children and adolescents have type 1 diabetes worldwide. IDF estimates 366 million diabetes cases by 2030. In Africa; an estimated 147 million adults are affected. In Nigeria there is an estimated burden of about 8.4 million diabetic patients. Nigeria has the highest number of diabetic cases in Sub-Saharan Africa with the prevalence rate of 1.5 million cases. (Diabetes Atlas 2017 \& WHO 2005).

Diabetes is the leading cause of non-traumatic amputation and blindness in working age adults; third leading cause of death among non-communicable diseases (NCDs). Its complications affect well-being and quality of life (QOL) of the individual negatively (Smeltzer, Bare, Hinkle \& Cheever, 2010).

WHO QoL group 1998, refers to the individual's perception of their position in life in the contexts of culture and value system in which they live and in relation to their goals, expectations, standards and concerns. Health Related Quality of life (HRQOL) refers to aspects of functioning directly related to diseases and medical treatment. Researchers have reported lower HRQOL in people with diabetes (DM) than for non-DMs. In Nigeria, the HRQOL of diabetics has been understudied (Odili, Ugboka \& Oparah, 2010).

\section{Purpose or need for study}

The purpose of this study is to assess and compare the Health- Related Quality of Life (HRQOL) of diabetic patients and non-diabetic patients in Cross River State, Nigeria. 


\section{Statement of the problem}

Globally, an estimated 425 million adults have diabetes worldwide with estimate of 366 million cases by 2030. In Nigeria about 1,707,000 Nigerians live with Diabetes Mellitus thereby imposing a major and most challenging health problem in the region.

Along with the increase in incidence of diabetes, both individual and societal expectations on the quality of life of persons living with diabetes have also increased. Taking control of diabetes to improve quality of life has put the spotlight on the need for additional support and education for patients with type 2 diabetes (Hossain, et. al.,2010). Studies showed that DM has a strong negative impact on the HRQOL. It became imperative to study the health-related quality of life of Diabetes Mellitus and nonDiabetic population in University of Calabar Teaching hospital.

\section{Specific objectives}

The specific objectives are to:

- Determine the Health -Related Quality of Life (HRQOL) scores of DM patients and that of nonDM using the WHOQOL-BREF.

- Compare the HRQOL scores of DM patients with that of non-DM

- Compare the HRQOL scores of DM patients with co-morbidities of those without

- Determine the influence of socio-demographic variables on the overall HRQOL mean score of the DM patients.

\section{Hypotheses}

- There will be no significant difference between the quality of life of people living with diabetes and those without diabetes

- Socio-demographic characteristics does not affect the quality of life of Diabetic patients

- Co-morbidities does not affect the quality of life of Diabetes patients

\section{Literature review}

Globally, 425 million adults have diabetes with 1 in 2 remain undiagnosed (IDF Atlas, 2017). World Health Organization (WHO, 2015) estimated that over four million Nigerians are living with type2 diabetes mellitus. Also, 40,815 deaths in adults due to diabetes were recorded.

Diabetes and its complications bring about substantial economic loss to people with diabetes and their families; health systems and national economies through direct medical costs and loss of work and wages.

Research indicates that coping skills and family stresses influence the management of diabetes. Depression is a serious co-occurring problem in diabetes that can affect glycaemic control (Glasgow et al., 1999). Combination of educational interventions to increasing knowledge, control, and self-efficacy among diabetics with behavioural psychosocial interventions (for example, enhancing coping skills and peer support) provide greater success in improving both metabolic outcomes and quality of life (Clement, 1995 Padgett et al., 1988).

Health-related quality of life (HRQoL) is a multi-dimensional concept that includes domains related to physical, mental, emotional and social functioning to measure population health, life expectancy, and causes of death, and focuses on the impact health status has on quality of life. A related concept of HRQoL is well-being, which assesses the positive aspects of a person's life, such as positive emotions and life satisfaction.

The World Health Organization Quality of Life (WHOQOL-BREF) instrument comprises 26 items, which measure four domains: physical, psychological, social relationships and environment. Sometimes a combination of generic and disease-specific HRQOL measures may be more appropriate for monitoring changes in a patient's health status due to an intervention (Chen T.H, Li L \& Kochen MM. 2005). 


\section{Methodology}

\section{Study type}

It is a descriptive cross-sectional study.

\section{Study setting}

The study was conducted at University of Calabar Teaching hospital (UCTH), a high patients volume hospital and referral research centre to other hospitals in the state.

\section{Study population}

The study population were diabetics and non-diabetics patients attending clinic at the UCTH.

\section{Sample size calculation for respondents}

Sample size of 200 for each group was determined using power analysis.

The sample size was calculated using sample size calculator.Net

- Confidence level $=95 \%$ and $80 \%$ power

- Confidence Interval = 5\%

- Population proportion $=50 \%$

- Population size $=412$ patients

The calculated sample size was 200 patients

\section{Sampling technique}

Purposive and convenient sampling procedure was applied in the selection of respondents based on inclusion criteria. All diabetes respondents were medically diagnosed patients while non-diabetes respondents were general outpatient and inpatient's in same hospital with no history of hypertension, stroke, arthritis, duodenal/gastric ulcers, cancers, HIV/AIDS and asthma.

\section{Sampling procedure}

The DM patients were recruited and interviewed during their clinic check-ups while non- diabetics were interviewed at GOPD and inpatients wards. The questionnaire was interviewer administered.

\section{Data collection and analysis}

Data collection lasted for three months. Instrument used for data collection was WHOQOL-BREF (28 items) with 12 additional questions soliciting demographic and clinical data of the respondents. Instrument were pre-tested for validity and viability.

Data analysis used Statistical Package for Social Sciences (SPSS) software. Summarized in proportions and mean scores. Hypotheses tested using Chi-square, student t-test and analysis of variance (ANOVA) at a significant level of $\mathrm{P} \leq 0.05$. Health related quality of life were measured on 5-point Likert scale with higher score indicating better quality of life.

\section{Ethical considerations}

Ethical approval was obtained from hospital, state ministry of health ethical committee and respondents. 
DOI: $10.21522 / \mathrm{TIJPH} .2013 .06 .03$. Art015

ISSN: $2520-3134$

\section{Results}

Table 1. Demographic characteristics of respondents, $(n=200)$

\begin{tabular}{|c|c|c|}
\hline Variable & Subcategory & $\begin{array}{l}\text { Percentage } \\
(\%)\end{array}$ \\
\hline Sex & $\begin{array}{l}\text { Female } \\
\text { Male }\end{array}$ & $\begin{array}{l}104(52 \%) \\
96(48 \%)\end{array}$ \\
\hline Marital status & $\begin{array}{l}\text { Married } \\
\text { Single } \\
\text { Divorced/separated/widowed }\end{array}$ & $\begin{array}{l}161(80.5 \%) \\
12(6 \%) \\
27(13.5 \%) \\
\end{array}$ \\
\hline Religion & Christianity & 100 \\
\hline Median Age & $\begin{array}{l}50-59 \mathrm{yrs} ;(35 \%) \\
60 \mathrm{yrs}+(65 \%)\end{array}$ & $53.9 \mathrm{yrs}$ \\
\hline $\begin{array}{l}\text { Median years living } \\
\text { with diabetes }\end{array}$ & & $8.27 \mathrm{yrs} \pm 6.45$ \\
\hline \multirow[t]{3}{*}{ Occupation } & Salary paid jobs & $102(51 \%)$ \\
\hline & Self employed & $61(30.5 \%)$ \\
\hline & Unemployed & $37(18,5 \%)$ \\
\hline $\begin{array}{l}\text { Level of } \\
\text { Education }\end{array}$ & $\begin{array}{l}\text { Did not go to school } \\
\text { Primary } \\
\text { Secondary } \\
\text { Post-Secondary (Diploma } \\
\text { \&Degree) }\end{array}$ & $\begin{array}{l}1(0,5 \%) \\
35(17.5 \%) \\
48(24 \%) \\
116(58 \%)\end{array}$ \\
\hline
\end{tabular}

Respondents were all Christians; predominantly married (81.8\%). more females (52\%) than males $(48 \%)$. Mean age 53.9yrs was modal age $=50-59 \mathrm{yrs} ;(35 \%)$; 50yrs+ $(68.5 \%)$. Median years living with diabetes 8.27 years. Majority (58\%) had tertiary education. Those on salary paid jobs were $(54.5 \%)$. Mean duration of years with diabetes 8.27yrs \pm 6.45 . No significant difference between demographic variable of diabetics and non-diabetics $(\mathrm{p}>0.05)$.

Table 2. Clinical data of diabetic respondents' $(n=200)$

\begin{tabular}{|l|l|l|}
\hline Variable & $\begin{array}{l}\text { No of } \\
\text { Respondents }\end{array}$ & Percentages \\
\hline Co-morbidities: & 141 & 70.5 \\
Yes & 59 & 29.5 \\
No & 4 & \\
\hline Co-morbidity Diseases Reported (n=141) & 80 & 2 \\
Asthma & 3 & 40 \\
& 30 & 1.5 \\
Hypertension & 1 & 15 \\
Congestive cardiac failure & 11 & 0.5 \\
& 1 & 5.5 \\
Arthritis & 11 & 0.5 \\
Pulmonary tuberculosis & $\mathbf{1 4 1}$ & 5.5 \\
Ulcer (Duodenal and stomach) & $\mathbf{7 1 \%}$ \\
\hline
\end{tabular}




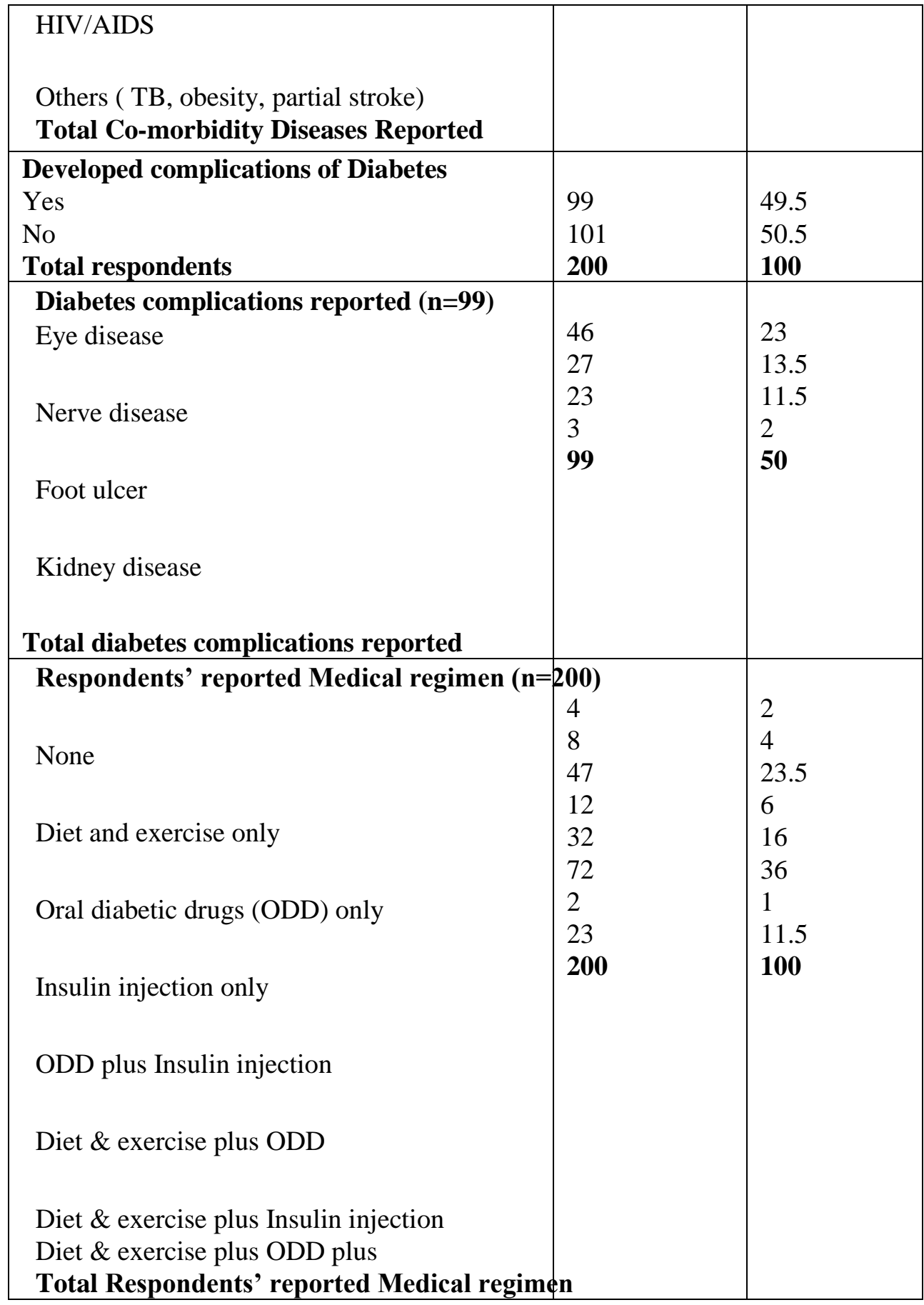

Clinical data of diabetic respondents showed that 70.5 percent $(n=141)$ were living with other disease while 29.5 percent $n=59$ ) of diabetic responds were not living with other diseases.

Diseases mostly exhibited by diabetic patients were hypertension 40 percent $(\mathrm{n}=80)$ and arthritis 15 percent $(n=30)$.

Diabetic disease related complications developed by diabetic respondents were approximately fifty percent $(n=99)$. Such diseases as nerve disease fourteen percent $(n=27)$, foot ulcer eleven percent $(n=23)$, kidney disease two percent (n-3) with eye disease ranking higher with twenty-three percent $(\mathrm{n}=46)$.

The responses revealed that the medical regimen mostly used by diabetic patients were diet, exercise plus oral diabetic drugs thirty-six percent $(n=72)$; oral diabetic drugs (ODD) only twenty three and half percent $(n=47)$; ODD plus Insulin injection sixteen percent $(n=32)$; diet, exercise and ODD plus eleven percent $(n=23)$; insulin injection only six percent $(n=12)$ with the least use of diet, exercise plus Insulin injection of one percent. 
DOI: $10.21522 / \mathrm{TIJPH} .2013 .06 .03$.Art015

ISSN: $2520-3134$

Table 3. Comparison of Respondents' mean scores differences on overall quality of life and satisfaction with health $(n=200)$

\begin{tabular}{|l|l|l|l|l|l|}
\hline Parameters & Groups & \multicolumn{2}{l|}{ t=test } \\
\hline & $\begin{array}{l}\text { Diabetics } \\
(\mathbf{n = 2 0 0})\end{array}$ & $\begin{array}{l}\text { Non-DM } \\
(\mathbf{n = 2 0 0})\end{array}$ & t-test & df & P-value \\
\hline $\begin{array}{l}\text { Overall quality of life } \\
\text { Satisfaction with health }\end{array}$ & $3.80 \pm 0.96$ & $4.14 \pm 0.58$ & -4.365 & 398 & $0.000^{*}$ \\
& $3.51 \pm 1.03$ & $4.09 \pm 0.58$ & -6.935 & 398 & $0.000^{*}$ \\
& & & & & \\
\hline Co-morbidities & & & & & \\
\hline Overall quality of life & $3.70 \pm 0.96$ & $3.88 \pm 0.89$ & -1.357 & 198 & 0.176 \\
\hline Satisfaction with health & $3.35 \pm 1.05$ & $3.65 \pm 1.00$ & -2.068 & 198 & $0.040^{*}$ \\
\hline
\end{tabular}

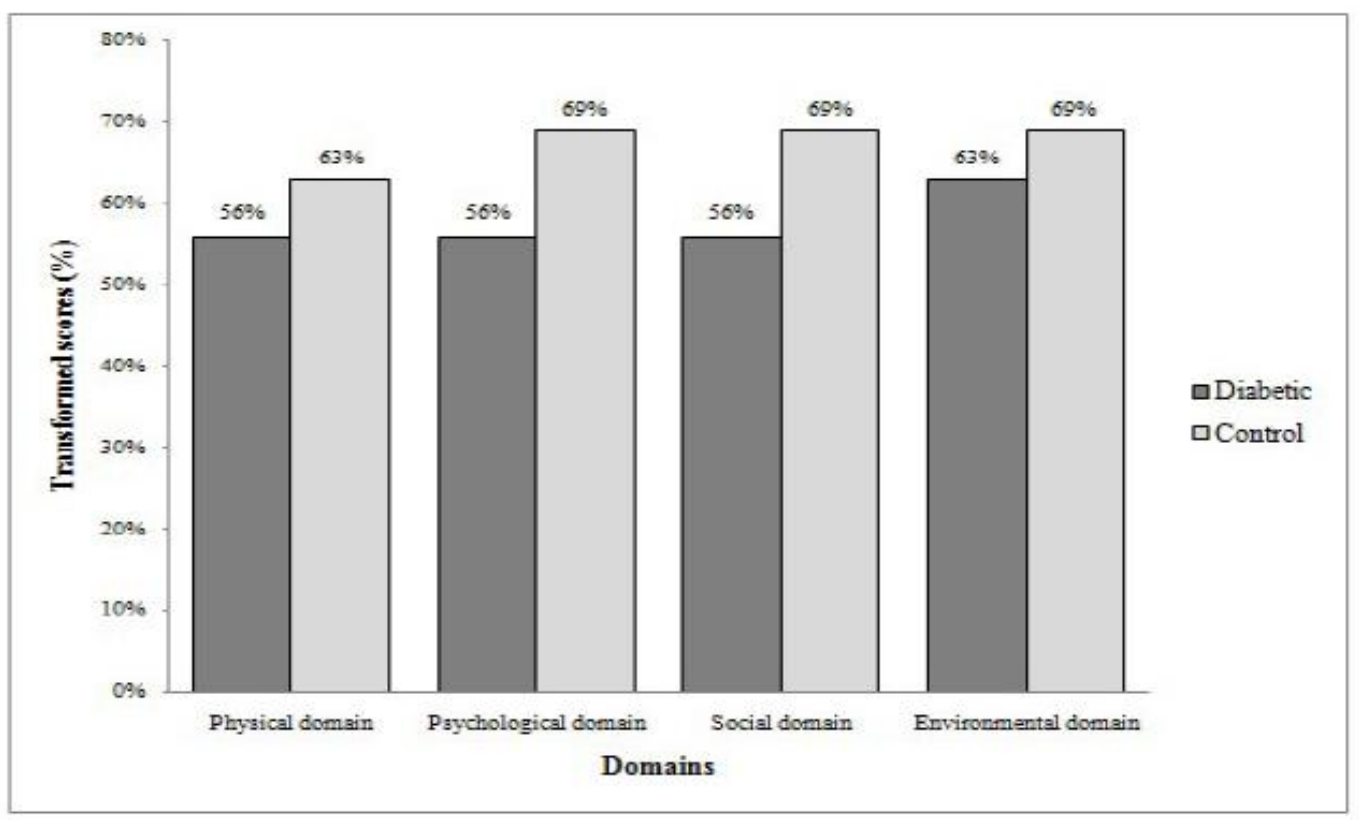

Figure 1. Bar charts showing the trasformed scores of HRQOL for Diabetic and Non-diabetic groups

Table 4. Comparison of mean scores differences in Four WHOQOL-BREF domains between diabetics and nondiabetics

\begin{tabular}{|c|c|c|c|c|c|}
\hline \multirow[t]{2}{*}{ Parameters } & \multicolumn{2}{|l|}{ Groups } & \multicolumn{3}{|l|}{ t-test } \\
\hline & $\begin{array}{l}\text { Diabetic } \\
(n=200)\end{array}$ & $\begin{array}{l}\text { Non-DM } \\
(n=200)\end{array}$ & t-test & df & P-value \\
\hline Physical domain & $23.17 \pm 3.39$ & $24.17 \pm 2.42$ & -3.398 & 398 & $0.001 *$ \\
\hline $\begin{array}{l}\text { Psychological } \\
\text { domain }\end{array}$ & $20.06 \pm 3.32$ & $21.53 \pm 2.51$ & -5.001 & 398 & $0.000^{*}$ \\
\hline Social domain & $10.20 \pm 2.47$ & $11.43 \pm 1.87$ & -5.640 & 398 & $0.000^{*}$ \\
\hline $\begin{array}{l}\text { Environmental } \\
\text { domain }\end{array}$ & $28.00 \pm 5.15$ & $\begin{array}{l}28.68 \pm 5.04 \\
4\end{array}$ & -1.325 & 398 & 0.186 \\
\hline Co-morbidities & Yes & No & & & \\
\hline Physical domain & $22.73 \pm 3.30$ & $23.55 \pm 3.43$ & -1.711 & 198 & 0.089 \\
\hline $\begin{array}{l}\text { Psychological } \\
\text { domain }\end{array}$ & $19.67 \pm 3.08$ & $20.39 \pm 3.48$ & -1.525 & 198 & 0.129 \\
\hline Social domain & $9.96 \pm 2.28$ & $10.40 \pm 2.62$ & -1.261 & 198 & 0.209 \\
\hline $\begin{array}{l}\text { Environmental } \\
\text { domain }\end{array}$ & $27.41 \pm 4.98$ & $28.50 \pm 5.25$ & -1.494 & 198 & 0.137 \\
\hline
\end{tabular}


Texila International Journal of Public Health Volume 6, Issue 3, Sep 2018

Table 5a. Comparison of mean scores differences in all the items of WHOQOL-BREF sub-scales for diabetics and non-diabetic groups

\begin{tabular}{|l|l|l|l|l|l|}
\hline Parameters & \multicolumn{2}{|l|}{ Groups } & \multicolumn{2}{l|}{$\begin{array}{l}\text { t-test for equality of } \\
\text { Mean: }\end{array}$} \\
\hline & $\begin{array}{l}\text { Diabeti } \\
\mathbf{c} \\
(\mathbf{n = 2 0 0} \\
)\end{array}$ & $\begin{array}{l}\text { Non-DM } \\
(\mathbf{n = 2 0 0})\end{array}$ & t-test & df & P-value \\
\hline $\begin{array}{l}\text { 1. Physical domain } \mathbf{( 7} \\
\text { items }\end{array}$ & $\begin{array}{l}3.42 \pm 0 . \\
96\end{array}$ & $3.99 \pm 0.68$ & -6.851 & 398 & $0.000^{*}$ \\
\hline $\begin{array}{l}\text { Activities of daily } \\
\text { living }\end{array}$ & $\begin{array}{l}3.33 \pm 0 . \\
93\end{array}$ & $2.31 \pm 1.00$ & 10.622 & 398 & $0.000^{*}$ \\
\hline $\begin{array}{l}\text { Dependence on } \\
\text { medicine \& medical } \\
\text { aids }\end{array}$ & $\begin{array}{l}3.23 \pm 0 . \\
96\end{array}$ & $3.83 \pm 0.73$ & -7.033 & 398 & $0.000^{*}$ \\
\hline Energy and fatigue & $\begin{array}{l}3.64 \pm 0 . \\
95\end{array}$ & $\begin{array}{l}3.00 \pm 0.64 \\
95\end{array}$ & -4.453 & 398 & $0.000^{*}$ \\
\hline Mobility & $\begin{array}{l}2.57 \pm 1 . \\
09\end{array}$ & $2.11 \pm 0.91$ & 4.574 & 398 & $0.000^{*}$ \\
\hline Pain and discomfort & $\begin{array}{l}3.70 \pm 0 . \\
97\end{array}$ & $4.02 \pm 0.72$ & -3.684 & 398 & $0.000^{*}$ \\
\hline Sleep and rest & $\begin{array}{l}3.29 \pm 1 . \\
04\end{array}$ & $3.93 \pm 0.69$ & -7.268 & 398 & $0.000^{*}$ \\
\hline Work capacity & & & -4.453 & 398 & $0.000^{*}$ \\
\hline
\end{tabular}

Table 5b. Comparison of mean scores differences in all the items of WHOQOL-BREF sub-scales for diabetics and non-diabetic groups

\begin{tabular}{|l|l|l|l|l|l|}
\hline Parameters & \multicolumn{3}{|l|}{ Groups } & \multicolumn{3}{l|}{ t-test for equality of Mean: } \\
\hline $\begin{array}{l}\text { 2, Psychological } \\
\text { domain (6 items) }\end{array}$ & $\begin{array}{l}\text { Diabetic } \\
(\mathbf{n = 2 0 0 )}\end{array}$ & $\begin{array}{l}\text { Non-DM } \\
(\mathbf{n = 2 0 0})\end{array}$ & t-test & df & P-value \\
\hline $\begin{array}{l}\text { Bodily image and } \\
\text { appearance }\end{array}$ & $3.39 \pm 1.03$ & $3.93 \pm 0.75$ & -6.037 & 398 & $0.000^{*}$ \\
\hline Negative feelings & $2.37 \pm 0.99$ & $2.22 \pm 0.71$ & 1.745 & 398 & 0.082 \\
\hline Positive feelings & $3.30 \pm 0.87$ & $3.54 \pm 0.69$ & -2.991 & 398 & $0.000^{*}$ \\
\hline Self-esteem & $3.39 \pm 1.03$ & $3.93 \pm 0.75$ & -6.037 & 398 & $0.000^{*}$ \\
\hline $\begin{array}{l}\text { Spirituality/Religion/P } \\
\text { ersonal beliefs }\end{array}$ & $2.37 \pm 0.99$ & $2.22 \pm 0.71$ & 1.745 & 398 & 0.082 \\
\hline $\begin{array}{l}\text { Thinking, learning, } \\
\text { memory and } \\
\text { concentration }\end{array}$ & $3.30 \pm 0.87$ & $3.54 \pm 0.69$ & -2.991 & 398 & $0.000^{*}$ \\
\hline
\end{tabular}

Table 5c. Comparison of mean scores differences in all the items of WHOQOL-BREF sub-scales for diabetics and non-diabetic groups

\begin{tabular}{|l|l|l|l|l|l|}
\hline Parameters & Groups & \multicolumn{3}{|l|}{ t-test for equality of Mean: } \\
\hline $\begin{array}{l}\text { 3. Social } \\
\text { domain (3) }\end{array}$ & $\begin{array}{l}\text { Diabetic } \\
(\mathbf{n = 2 0 0 )}\end{array}$ & $\begin{array}{l}\text { Non-DM } \\
(\mathbf{n = 2 0 0 )}\end{array}$ & t-test & df & P-value \\
\hline $\begin{array}{l}\text { Personal } \\
\text { relationships }\end{array}$ & $3.75 \pm 0.91$ & $4.05 \pm 0.61$ & -3.871 & 398 & $0.000^{*}$ \\
\hline Social support & $2.99 \pm 1.30$ & $3.77 \pm 1.00$ & -6.691 & 398 & $0.000^{*}$ \\
\hline
\end{tabular}


DOI: $10.21522 /$ TIJPH.2013.06.03.Art015

ISSN: $2520-3134$

\begin{tabular}{|l|l|l|l|l|l|}
\hline $\begin{array}{l}\text { Sexual life } \\
\text { satisfaction }\end{array}$ & $3.46 \pm 1.05$ & $3.62 \pm 0.83$ & -1.694 & 398 & 0.091 \\
\hline
\end{tabular}

Table 5e. Comparison of mean scores differences in all the items of WHOQOL-BREF sub-scales for diabetics and non-diabetic groups

\begin{tabular}{|c|c|c|c|c|c|}
\hline Parameters & \multicolumn{2}{|l|}{ Groups } & \multicolumn{3}{|l|}{ t-test } \\
\hline $\begin{array}{l}4 . \\
\text { Environmental } \\
\text { domain } \\
\text { items) }\end{array}$ & $\begin{array}{l}\text { Diabetic } \\
(n=200)\end{array}$ & $\begin{array}{l}\text { Non- } \\
\text { DM } \\
(\mathbf{n}=\mathbf{2 0 0})\end{array}$ & t-test & df & $\begin{array}{r}P \text { - } \\
\text { value }\end{array}$ \\
\hline $\begin{array}{l}\text { Financial } \\
\text { resources }\end{array}$ & $2.99 \pm 0.99$ & $6^{3.21 \pm 0.8}$ & -2.368 & 398 & $0.018^{*}$ \\
\hline $\begin{array}{l}\text { Freedom, } \\
\text { physical safety } \\
\text { and security }\end{array}$ & $3.77 \pm 0.78$ & $6^{3.89 \pm 0.6}$ & -1.664 & 398 & 0.097 \\
\hline $\begin{array}{l}\text { Health and } \\
\text { social care: } \\
\text { accessibility and } \\
\text { quality }\end{array}$ & $3.67 \pm 0.92$ & $0^{3.77 \pm 0.8}$ & -1.218 & 398 & 0.224 \\
\hline $\begin{array}{c}\text { Home } \\
\text { environment }\end{array}$ & $3.84 \pm 0.90$ & $9^{3.87 \pm 0.7}$ & -0.296 & 398 & 0.768 \\
\hline $\begin{array}{l}\text { Opportunities } \\
\text { for acquiring } \\
\text { new information } \\
\text { and skills }\end{array}$ & $3.49 \pm 0.94$ & $2^{3.40 \pm 0.8}$ & 0.963 & 398 & 0.336 \\
\hline $\begin{array}{l}\text { Participation } \\
\text { in } \quad \text { and } \\
\text { opportunities for } \\
\text { recreation/leisur } \\
\text { e activities }\end{array}$ & $3.02 \pm 1.02$ & $0^{3.06 \pm 0.9}$ & -0.415 & 398 & 0.678 \\
\hline $\begin{array}{l}\text { Physical } \\
\text { environment } \\
\text { (pollution/noise/ } \\
\text { traffic/climate) }\end{array}$ & $3.87 \pm 0.76$ & $6^{3.96 \pm 0.8}$ & -0.454 & 398 & 0.650 \\
\hline Transport & $3.37 \pm 1.13$ & $5^{3.53 \pm 0.9}$ & -1.487 & 398 & 0.138 \\
\hline
\end{tabular}

Table 6. Analysis of Variance in mean scores between socio-demographic and overall HRQOL scores of diabetic groups

\begin{tabular}{|l|l|l|l|l|l|}
\hline $\begin{array}{l}\text { Socio } \\
\text { demographic }\end{array}$ & Parameter & $\begin{array}{l}\text { Mean + } \\
\text { SD }\end{array}$ & $\begin{array}{l}\text { t-test } \\
\text { ANOVA }\end{array}$ & df & P-value \\
\hline Sex & $\begin{array}{l}\text { Female } \\
(\mathrm{n}=104)\end{array}$ & $3.88 \pm 0.95$ & 1.380 & 198 & 0.169 \\
\hline & Male (n=96) & $3.70 \pm 0.96$ & & & \\
\hline $\begin{array}{l}\text { Level of } \\
\text { education }\end{array}$ & $\begin{array}{l}\text { Did not go to } \\
\text { school (n=1) }\end{array}$ & 5.00 & 3.775 & 3.196 & $0.012^{*}$ \\
\hline & $\begin{array}{l}\text { Primary Six } \\
(\mathrm{n}=35)\end{array}$ & $3.37 \pm 1.06$ & & & \\
\hline & $\begin{array}{l}\text { Secondary } \\
(\mathrm{n}=48)\end{array}$ & $3.75 \pm 1.12$ & & & \\
\hline & $\begin{array}{l}\text { Post } \\
\text { Secondary } \\
(\mathrm{n}=116)\end{array}$ & $3.93 \pm 0.81$ & & & \\
\hline
\end{tabular}




\begin{tabular}{|l|l|l|l|l|l|}
\hline Occupation & $\begin{array}{l}\text { Employed in } \\
\text { salary } \\
\text { job(n=102) }\end{array}$ & $3.89 \pm 0.88$ & & & \\
\hline & $\begin{array}{l}\text { Self- } \\
\text { employed } \\
(\mathrm{n}=61)\end{array}$ & $3.64 \pm 1.05$ & 1.337 & 2,197 & 0.265 \\
\hline & $\begin{array}{l}\text { Unemployed } \\
(\mathrm{n}=37)\end{array}$ & $3.78 \pm 1.00$ & & & \\
\hline Marital Status & Single (n=12) & $3.83 \pm 1.19$ & & & 0.731 \\
\hline & $\begin{array}{l}\text { Married } \\
(\mathrm{n}=161)\end{array}$ & $3.77 \pm 0.92$ & 0.314 & 2,197 & \\
\hline & $\begin{array}{l}\text { Divorced/Sep } \\
\text { arated/Wido } \\
\text { wed(n=27) }\end{array}$ & $3.93 \pm 1.07$ & & & \\
\hline
\end{tabular}

\section{Discussion}

Mean scores presented on Tables $5 \mathrm{a}-5 \mathrm{e}$ showed that diabetes had lower mean scores and reported lower overall health related quality of life (HRQOL) showing dissatisfaction in all the four domains of WHOQOL-BREF.

The mean scores differences were all statistically significant except in the: i) environmental domain. ii) Spirituality/Religion/Personal belief and negative feelings items in psychological domain. (iii) Satisfaction with sexual life in the social domain and iv) Between the diabetics with and without comorbidities.

Odili et al (2010) found lower HRQOL among diabetics than the non-diabetic group in all the domains with the largest difference in the physical, psychological and social domains.

In current study 99(50\%) of the respondents developed diabetic complications which could have impacted negatively on their domains resulting in reduced HRQOL.

Patients with diabetes had a significant lower mean score than the non-diabetics in personal relationship and social support $(\mathrm{P}=0.000)$. Current study showed lower satisfaction with sexual life among diabetics than the non-diabetic group $(\mathrm{P}=0.091)$. Odili et al (2010) reported on poor sexual activities among diabetic patients.

Environmental domain analysis showed that only financial resources had significant difference between the two groups. All other factors in environmental domain except financial resources were impacted upon by diabetes disease. There were lower mean scores in HRQOL in all the domains among diabetics with co-morbidities than those without co-morbidities.

Diabetics with higher education and better paid job had a significant higher mean score than the less educated $(\mathrm{p}<0.05)$. reported

\section{Limitations and strenghts}

The studied patients received care in tertiary hospital, whose conditions were likely to be under control. Worse HRQOL might be reported by respondents who were not accessing specialist care. World Health Organization quality of life instrument is a generic tool which has lower sensitivity to elicit symptoms as disease specific instrument. This limit the generalization of findings.

\section{Recommendations}

Awareness creation on spread of diabetic disease and the provision of appropriate education on selfcare management to the society will reduce complications associated with diabetes.

Diabetic care team (clinicians and family members) and policy makers should implement appropriate strategies to improve quality of life of diabetic patients.

Further research with wider population using disease specific instrument is recommended for wider generalization of findings. 
DOI: $10.21522 / \mathrm{TIJPH} .2013 .06 .03$. Art015

ISSN: $2520-3134$

\section{Conclusion}

Diabetes impacts negatively (mostly on physical domain) on the health-related quality of life of diabetics when compared with non-diabetic persons in Cross Rivers State of Nigeria. Financial resource is one environmental domain factor that significantly impacted upon by diabetes and its management. Diabetics need support, both tangible and intangible to enable them cope with their health problem. Awareness creation on the occurrence, prevention and management of diabetes will reduce the prevalent of diabetes disease in Nigeria. World Health Organization quality of life (WHOQOL-BREF) generic tool lowers sensitivity to elicit symptoms as disease specific instrument thereby limiting the generalization of findings and comparison of the results obtained. Studying comparison group, with the same hospital became a major strength which enabled the researcher to identify the magnitude of the impact of the disease and its management on the respondents' HRQOL in each domain.

\section{Reference}

[1].Clement S. Diabetes self-management education. Diabetes Care. 1995;18:1204-1214. [PubMed].

[2].Chen T.H, Li L \& Kochen MM. 2005. A systematic review: How to choose appropriate health-related quality of life (HRQOL) measures in routine general practice? J Zhejiang Univ Sci B. 2005 Sep;6(9):936-940.

[3].Hossain MA, et al 2010. Diabetes and Ramadan

[4].IDF Diabetes Atlas. Global estimates of the prevalence of diabetes for 2011 and 2030. $8^{\text {th }}$ Edition 2017.

[5].Odidi V., Ugboka L.U., Oparah A. C. 2010. Quality of life of people with diabetes in Benin city as measured with WHOQOL-BREF. The Internet Journal of Law, Healthcare and Ethics. 2008 Volume 6 Number 2.

[6].Padgett D, Mumford E, Hynes M, Carter R. Meta-analysis of the effects of educational and psychosocial interventions on management of diabetes mellitus. Journal of Clinical Epidemiology. 1988; 41:1007-1030. [PubMed]

[7].Suzanne C. et al., 2010. Brunner and Suddarth's Textbook of Medical-Surgical Nursing (Two Volume Set). Twelfth edition.

[8].WHO. Development of the WHOQOL-BREF quality of life assessment. Psychological Medicine. 1998 May; 28(3):551-8. 